\title{
Does any therapy really work for neurocardiogenic syncope?
}

\author{
Nathaniel M. Hawkins, Andrew D. Krahn \\ Division of Cardiology, University of British Columbia, Vancouver, Canada
}

\begin{abstract}
Effectiveness of a treatment for neurocardiogenic syncope can be defined in terms of symptom response, quality-of-life, healthcare utilization, treatment side effects and cost-effectiveness. Most trials have focused on syncope recurrence or burden, without assessing quality-of-life formally. Drug and device interventions are characterized by a dearth of randomized controlled trials, with those few of robust design demonstrating little impact on recurrence of syncope. General advice includes hydration, trigger recognition and counter pressure maneuvers to attenuate episodes. Lifestyle recommendations have limited comparative effectiveness evidence, but are favored due to lack of side effects and low cost. The frequency of syncope improves in many patients regardless of the intervention, although ultimate recurrence of syncope remains high. In the minority of patients seeking treatment due to recurrence, midodrine has reasonable supporting evidence for effectiveness with some evidence for beta-blockers in older age patients. Emerging evidence favors pacing in patients with asystole during spontaneous (as opposed to provoked) syncope. Combining long-term implantable cardiac monitoring, tilt and adenosine triphosphate testing may yet accurately define the optimal minority who benefit from pacing. In the remaining majority, pharmacologic and device interventions should be used sparingly until clear benefits are established. Better understanding of patient fears, beliefs and behaviors may help develop cognitive therapies and improve quality-of-life alongside the focus on physical symptoms. (Cardiol J 2014; 21, 6: 616-624)
\end{abstract}

Keywords: syncope, drug therapy, placebo

\section{Introduction}

The question of how to approach therapy for neurocardiogenic syncope (NCS) does not come with a simple answer. In effect, a subgroup of patients with NCS seek help from their physicians for a recurrent, troubling and, at times, life-altering condition that many patients either minimize or even fail to report. In so doing, they declare themselves as treatment seeking, fundamental to the journey to clinical improvement regardless of the intervention. Along come physiologists who seek to understand dynamic alterations in blood pres- sure regulation and cerebral perfusion, and who propose deductive interventions that "should help" because of presumed knowledge of the underlying mechanism of the faint. Thus, doctors prescribe thoughtful therapies, patients want to get better, and syncope generally improves or resolves.

This apparently effective empiric approach collides with systematic comparative effectiveness research methodology, which shows that most patients get better (i.e. syncope does not recur) regardless of intervention, including placebo, with trends to small incremental improvement with a small proportion of the many proposed agents or

Address for correspondence: Dr Andrew Krahn, Arrhythmia Service, $9^{\text {th }}$ Floor, Gordon and Leslie Diamond Health Care Center, 2775 Laurel Street, Vancouver, BC, Canada V5Z 1M9, tel: 1-604-875-4111 ext. 69821, fax: 1-604-875-5504, e-mail:akrahn@mail.ubc.ca

Received: 09.09.2014 Accepted: 20.10.2014 
Table 1. Treatment effect size in randomized trials of drug and device therapy for neurocardiogenic syncope in the Cochrane Library meta-analysis.

\begin{tabular}{|c|c|c|c|}
\hline \multirow[t]{2}{*}{ Outcome } & $\begin{array}{l}\text { Meta-analysis } \\
\text { randomized } \\
\text { (Cochrane) [4] }\end{array}$ & $\begin{array}{l}\text { Meta-analysis } \\
\text { randomized } \\
\text { (Cochrane) [4] }\end{array}$ & \multirow{2}{*}{$\begin{array}{l}\text { Meta-analysis } \\
\text { randomized } \\
\text { or non-randomized } \\
\text { (Vyas et al.) [5] }\end{array}$} \\
\hline & $\begin{array}{l}\text { Standard } \\
\text { treatment }\end{array}$ & $\begin{array}{l}\text { Placebo or non-active } \\
\text { pacemaker }\end{array}$ & \\
\hline \multicolumn{4}{|l|}{ Beta-blockers } \\
\hline Syncope recurrence & $0.36(0.21-0.62)$ & $1.07(0.80-1.44)$ & $0.48(0.22-1.04)$ \\
\hline Provocation syncope & $0.75(0.58-0.97)$ & $\begin{array}{c}0.32(0.14-0.73) \text { parallel } \\
0.87(0.31-2.43) \text { cross }\end{array}$ & - \\
\hline \multicolumn{4}{|l|}{ Alpha-adrenergic } \\
\hline Syncope recurrence & - & - & $\begin{array}{c}0.35(0.04-2.88) \text { etilefrine } \\
0.12(0.05-0.26) \text { midodrine }\end{array}$ \\
\hline Provocation syncope & - & $\begin{array}{c}0.94(0.59-1.48) \text { etilefrine } \\
0.12(0.04-0.36) \text { midodrine }\end{array}$ & - \\
\hline \multicolumn{4}{|l|}{$\begin{array}{l}\text { Selective serotonin } \\
\text { reuptake inhibitors }\end{array}$} \\
\hline Syncope recurrence & - & $0.39(0.20-0.76)$ & $0.28(0.10-0.74)$ randomized \\
\hline Provocation syncope & - & $0.75(0.51-1.11)$ & - \\
\hline \multicolumn{4}{|l|}{ Tilt training } \\
\hline Syncope recurrence & - & - & $\begin{array}{c}\mathbf{0 . 3 0}(\mathbf{0 . 1 5}-\mathbf{0 . 6 1}) \\
0.47(0.21-1.05) \text { randomized }\end{array}$ \\
\hline \multicolumn{4}{|l|}{ DDD pacemaker } \\
\hline Syncope recurrence & $0.20(0.10-0.40)$ & $0.89(0.58-1.38)$ non-active & $0.45(0.09-2.14)$ non-active \\
\hline
\end{tabular}

interventions [1-3]. This chapter addresses the issue about whether or not any treatment for NCS really is effective.

\section{Effectiveness of drugs and devices in meta-analysis}

The short answer to the question: 'does anything work?' is 'no therapy has proven to be effective', at least according to the Cochrane meta-analysis of randomized parallel and cross-over trials of drug and pacemaker therapy for NCS (Table 1) [4]. A second recent meta-analysis confirmed the Cochrane findings, this time including non-randomized as well as randomized studies (Table 1) [5]. Taking only randomized studies with placebo or non-active (that is placebo) device, only selective serotonin reuptake inhibitors (SSRIs) and midodrine (but not etilefrine) decrease syncope recurrence.

However, the totality of evidence for SSRIs derives from 2 studies $(n=131)$ and for midodrine 4 studies $(n=136)$. Beta-blockers, tilt training, and pacing all failed to affect syncope recurrence in robustly designed trials, although many studies were arguably underpowered or projecting an ambitious relative risk reduction. Specifically in pacing, meta-analysis of 9 randomized trials revealed reduced risk of syncope in unblinded studies and those comparing pacemaker algorithms, but not in double-blinded trials [6].

The meta-analyses highlight the limitations of current evidence and inefficacy of current therapies. Effect size was markedly greater when comparing the intervention to standard treatment (usually non-pharmacological or other pharmacological therapy) as opposed to placebo or non-active device. Confidence intervals were wide even after aggregating studies, reflecting small patient numbers and variable treatment effect. All 3 meta-analyses detected significant heterogeneity. Finally, only 2 outcomes were consistently reported: syncope recurrence and syncope during provocation.

\section{Effectiveness and study design}

Study size and design overwhelmingly influence apparent effect size [7]. The NCS literature is characterized by small to medium sized studies 
with inadequate controls and conflicting results. Many factors have hindered the evolution of evidence: (1) Early studies predated widespread adoption of the multicenter randomized controlled trial; (2) Therapies are off-patent with limited industry support for trials; (3) The target population lacks a gold-standard diagnostic test.

The Cochrane assessment identified many sources of bias including randomization and sequence generation, allocation concealment, blinding, incomplete outcome data, and selective reporting. Small, non-randomized studies also increase susceptibility to publication bias. This is particularly true in conditions with infrequent outcomes, where a chance change in frequency of syncope may influence small studies. Possible bias was detected by funnel plot in the most recent meta-analysis [5].

Robust study design failed to support any therapy in NCS. The best beta-blocker evidence (or lack thereof) comes from the Prevention of Syncope Trial (POST), which randomized 208 patients with recurrent syncope and positive tilt table test to receive metoprolol or placebo [3]. No difference was observed in recurrence of syncope, even when stratified by age and isoproterenol response during tilt test. Subsequent pooled analysis with non-randomized patients found a significant interaction of beta-blockers with age, suggesting benefit after age 42 , and potential harm in younger patients [8].

Likewise, the Vasovagal Syncope International Study (VASIS) demonstrated no benefit to etilefrine in a randomized, double blind, placebo-controlled multicenter trial [9]. The second Vasovagal Pacemaker Study (VPS II) and vasovagal syncope and pacing trial (SYNPACE) contradicted the apparent efficacy of earlier non-blinded studies when patients received pacemakers with concealed randomization to active pacing or off $[1,2]$.

\section{Effectiveness of non-pharmacological treatment (and the placebo effect)}

Non-pharmacological interventions (education, increasing fluid and salt, physical counter-pressure maneuvers) are considered first-line treatment in guidelines due to sound physiologic rationale, simple implementation, and the absence of harm, side effects or additional cost [10]. Counselling focuses on educating the patient and the family to understand the condition, emphasizing the benign prognosis and trigger avoidance. Hydration and liberalizing salt intake improves orthostatic tolerance to tilt testing in small acute or short-term studies, increasing plasma volume, cerebrovascular and peripheral vascular control [11, 12]. No long-term, controlled evidence exists for any conservative treatment.

However, syncope recurrence decreases over time in cohorts without additional intervention (syncope is self-limited for the majority) [13, 14]. In 100 patients with NCS, the median number of syncopal recurrences was significantly lower in the first year after vs. before non-pharmacological treatment (median 0 vs. $3 ; \mathrm{p}<0.001$ ) [14]. A placebo effect, regression to the mean, and spontaneous improvement undoubtedly contribute. However, the principle precept of medical practice applies: first - do no harm. These low-risk lifestyle recommendations are therefore favored, with diagnosis and reassurance the cornerstones of management despite lack of comparative evidence to support their use.

Physical counter-pressure maneuvers (PCM) involve isometric contractions of legs or arms or squats during the patient's prodrome. Two initial studies demonstrated that arm tensing and leg crossing raised blood pressure $30 \%$ to $60 \%$ and postponed or prevented symptoms on tilt-table testing $[15,16]$. The subsequent Physical Counterpressure Maneuvers Trial (PC Trial) randomized 223 patients with NCS and prodromal symptoms to $\mathrm{PCM}$ or conventional therapy, blinding patients to randomization allocation. Despite a similar number of pre-syncopal episodes in each arm, fewer PCM patients progressed to syncope. The respective syncope recurrence rates were $31.6 \%$ vs. $50.9 \%$ over 14 months mean follow-up (RR 0.36; 95\% CI 0.11-0.53) [17]. The intervention requires compliance, which in itself promotes a placebo effect. However, the nature of the intervention prevents double blind investigation. Moreover, the beneficial effect of training is inherent to the treatment and equally useful whether 'placebo effect' or otherwise.

Syncopal frequency profoundly improves in the control arms of almost every study compared to baseline. This 'placebo effect' has three drivers of pre-to-post change: (1) Random events and measurement/recall error contribute to inherent variability; (2) The condition improves either spontaneously or in response to previous interventions e.g. education or reassurance; (3) The intervention or placebo imposes an additional effect above the inherent variability and evolving disease state.

The magnitude of this placebo effect is significant in NCS. For example, implanting a cardiac 
Table 2. Potential outcomes of interest when managing neurocardiogenic syncope.

\begin{tabular}{|c|c|c|}
\hline & Outcome & Comments \\
\hline Survival & Death & Not affected by neurocardiogenic syncope \\
\hline \multirow[t]{3}{*}{ Syncope recurrence } & Episodes per unit time & Optimal metric in relation to quality of life unknown \\
\hline & Proportion with recurrence & Association with quality of life poorly understood \\
\hline & Time to recurrence & \\
\hline \multirow[t]{4}{*}{ Quality of life } & Disease specific questionnaire & Standardized tools rarely used in trials to date \\
\hline & Generic questionnaire & \\
\hline & Disability/restriction & \\
\hline & Trauma/injury & \\
\hline \multirow[t]{3}{*}{ Treatment } & Adverse effects & Severity and time frame of assessment important \\
\hline & Compliance, discontinuation & Placebo effect powerful and inherent to therapy \\
\hline & Placebo effect & \\
\hline \multirow[t]{3}{*}{ Healthcare utilization } & Hospitalization & Patient and health system relevant outcomes \\
\hline & Emergency Department visit & Interaction with quality of life merits investigation \\
\hline & Clinic or medical contact & \\
\hline Economic & Cost-effectiveness & Requires standardized measure of health utility \\
\hline
\end{tabular}

monitor with no direct therapeutic efficacy reduces events. In 25 patients with severe vasovagal syncope (mean of $6.9 \pm 4.6$ episodes per year), only 12 experienced any syncope over 17 months post implantable cardiac monitor (ICM) [18]. The placebo effect appears greater for devices than drugs. First, syncope recurrence is reduced more in the placebo arms of pacemaker studies than drug trials. Second, the apparent treatment effect of pacemakers is greater than drugs in non-controlled trials (i.e. larger placebo effect). Finally, pacing is far superior to beta-blocker in a direct comparison, suggesting greater placebo effect given the lack of proven efficacy for either strategy [19].

\section{How is effectiveness defined?}

When considering: “does anything work?”, 'work' must be defined. From the patient perspective, patient relevant outcomes are defined as 'how a patient feels, functions or survives' [20]. These are divided into benefit outcomes and harm outcomes [21]. The former include 'hard endpoints' (death, hospitalization), symptoms and health-related quality-of-life (QOL). Since NCS does not affect survival, symptoms and QOL must be balanced against adverse effects. This balance involves weightings of complex multidimensional structures. Individual patients assign value differently to symptoms and domains of QOL. Both benefit and harm effects vary in magnitude depending on treatment, outcome assessed, time frame and population. For example, pacing in major registries conveys significant morbidity even in the short term. What improvement in QOL is worth trading for a lifetime risk of pacing complications, when not one of the NCS pacing trials reported any major injuries related to syncope?

No guideline or consensus document has specifically addressed how efficacy should be defined. Table 2 outlines potential outcomes. The Cochrane meta-analysis delineates a hierarchy of patient relevant outcomes: metrics of syncope are followed by QOL, incidence of physical trauma, and severity of side effects. Syncope induced by provocation occupies the final and least impactful position in the hierarchy.

Tilt testing has almost no value in assessing treatment efficacy [10]. Reproducibility varies up to $90 \%$ depending on the response [4]. Positive responses decrease with repeated testing irrespective of assigned treatment, with approximately $50 \%$ of patients responding to placebo [22]. With respect to QOL, remarkably few studies in the Cochrane meta-analysis reported any measure: 3 of 16 beta-blocker, 2 of 8 alpha-adrenergic agonist; 1 of 2 SSRI, and 1 of 9 pacing studies [4]. Only 3 of these studies employed a validated questionnaire, the remainder consisting of self-reported well-being and scales without validation. 


\section{How and why are patients impaired?}

Patients with NCS report worse QOL and experience higher levels of anxiety, depression and somatization disorders than control populations [23, 24]. The level of impairment is similar to severe arthritis, pain, epilepsy and other chronic disorders. In cohort studies both generic and disease specific questionnaires point to several key domains of impairment: fear, worry and embarrassment; depression and anxiety; activity impairment including mobility, driving, employment, and exercise; interference with relationships [25-28].

However, although syncope burden and QOL exhibit a dose-response relationship, the correlation coefficient is weak and most apparent in more severe cases [14, 23, 26]. Given the complex pathophysiology and precipitants, complete eradication of symptoms is unlikely in many patients. It is therefore likely that simply targeting reduction in syncope burden will have at best a modest effect on QOL.

Cognitive behavioral therapy addresses thoughts, beliefs and somatic attention that sustain disability and distress. Syncope sufferers have many therapeutic targets: perceived likelihood of fainting, negative consequences, and lack of control; unfounded activity restriction, avoidance and protective behavior; vigilance of somatic signs linked with fainting and amplification by fear arousal. In a small retrospective observational series of 9 patients, syncopal episodes and medical consultations significantly reduced following psychology intervention aiming to restructure maladaptive beliefs and somatic attention [29]. These preliminary findings merit further exploration in larger cohorts, but in some respects lack champions of the technique in the syncope realm.

\section{Is guided therapy effective?}

Lack of evidence from controlled clinical trials mandates reliance on personal experience and observations. Physician's "gestalt" is difficult to quantify and patient selection may improve response in select cases despite trial evidence to the contrary. Choices are limited when treating a patient. Nevertheless, we equally recognize the numerous alternate explanations for anecdotal response: placebo or expectation effect, attrition and follow-up bias, observer and recall bias, the play of chance or regression to the mean.

Tailoring therapy to individual patient physiology was for years the Rosetta Stone of NCS research, exemplified by the Vasovagal Syncope
International Study (VASIS) classification based on tilt testing response [30]. Subsequent randomized trials selecting patients with specific characteristics unequivocally refuted such approaches. However, the reliance on tilt testing to characterize phenotypes has arguably been the greatest inadvertent flaw in guided therapy strategies. Provocation induces a physiological response, not the actual event. Rhythm disturbance during provocation is common and correlates poorly with real-life spontaneous syncope [31, 32]. The sensitivity and specificity for events is accordingly low, preventing a homogeneous population being defined.

Beyond placebo effect, pacing can only be effective when syncope is secondary to asystole. Two studies, including the Second International Study on Syncope of Uncertain Etiology (ISSUE-2), examined reproducibility of electrocardiographic findings in NCS patients with an ICM and $\geq 2$ syncopal episodes $(n=12$ and $n=22)[18,33]$. The rhythm during first recurrence (abnormal or not) was almost identical in subsequent recurrences, although only a minority were actually attributable to bradyarrhythmia/asystole ( $n=1 / 12$ and $n=6 / 22$ ).

Nevertheless, the findings suggest ICMs may overcome 2 key limitations of tilt testing: reproducibility and association with spontaneous events. The International Study of Syncope of Uncertain Etiology (ISSUE-3) trial tested this hypothesis, randomizing 77 patients with purported neurally-mediated syncope and ICM documented asystole (mean $11 \mathrm{~s}$ ) to dual chamber pacing with rate-drop response or sensing only [34]. Respectively, $25 \%$ vs. $57 \%$ of patients had syncope recurrence at 2 years, a $57 \%$ risk reduction (95\% CI 4-81). Although promising, the many aforementioned caveats apply: patients were highly selected; risks outweighed benefits (5 pacing complications vs. no major adverse events secondary to syncope); high recurrence despite optimal treatment (25\%), and QOL was not assessed.

There is emerging evidence that adenosine triphosphate (ATP) hypersensitivity may guide pacemaker selection in refractory cases. In ISSUE-2 and smaller cohort studies, a positive ATP test failed to predict syncopal recurrence and had no correlation with ICM documented rhythm during spontaneous syncope [18, 31]. However, the recent single-blinded ATP Multicenter Study randomized 80 elderly patients (mean age $75.9 \pm$ \pm 7.7 years) with syncope of unknown origin and positive ATP test to active or backup pacing, with syncope recurred in $21 \%$ vs. $66 \%$, respectively (HR $0.25 ; 95 \%$ CI $0.12-0.56$ ) [35]. This trial strongly supports the use of the test in patients in whom 
pacing is contemplated, but uptake in practice and guidelines has been cautious. Differences in study populations and diagnostic criteria may partly explain the discrepancy with earlier cohort studies.

A final conundrum has emerged from subgroup analysis of ISSUE-3 [36]. In 136 NCS patients with implantable loop recorder (ILR) documented asystole, asystolic response during baseline tilt test had no diagnostic utility in predicting recurrence or electrocardiogram pattern during episodes. However, pacing only prevented syncope recurrence in patients with a previous negative tilt test. Pacing was ineffective in those with positive tilt tests despite subsequent documented asystole, the recurrence rate being similar to untreated patients and presumably reflecting concurrent vasodepressor response. This inverts previous indications for pacing and shifts tilt testing from a diagnostic to therapeutic guide. The challenge now becomes finding the optimal combination of ILR monitoring, tilt and ATP testing to accurately segregate the population most likely to benefit from pacing.

\section{What are realistic goals?}

The lifetime risk of syncope in the general population exceeds $30 \%$, with many experiencing recurrent symptoms [37]. For NCS, recurrencerates within 1 year typically approach one third of patients, even with optimal treatment in the most robust studies (Table 3). Pre-syncope is even more common, with $83 \%$ of patients in the PC trial and $96 \%$ in VPS II reporting symptoms [17]. Couple these outcomes with poorly understood mechanisms and confounding orthostatic and autonomic syndromes, and the target 'response' becomes even less certain. If we acknowledge the implausibility of widespread complete success, we must better understand how less frequent syncope and pre-syncope may become more acceptable to patients, in effect "debulking" the disease.

\section{What is effective for the population and health system?}

From a population perspective, guided therapy limits generalizability. In the major pacing trials $<5 \%$ of patients screened were eligible. Recruitment rates in multicenter trials highlight the same challenge. For example, the PC-trial of counterpressure maneuvers included recurrent vasovagal syncope with prodromal symptoms [17]. In 22 months, 223 patients were recruited in 15 centers (8.1 patients per center per year).

By contrast, ISSUE-3 mandated $\geq 3$ s syncopal asystole or $\geq 6 \mathrm{~s}$ asymptomatic asystole [34]. In 48 months, 77 patients were enrolled in 29 centers (0.7 patients per center per year). Obviously far fewer patients have recurrent NCS associated with significant asystole, limiting the population impact of the intervention.

From a health system or government perspective, a simple low-cost intervention with even mild symptomatic improvement is highly effective if medical contacts, Emergency Department (ED) attendances and hospitalizations are reduced. The patients with refractory symptoms attending specialized services in the aforementioned trials represent the tip of the iceberg. For example, the crude cumulative rate of syncope events in the ED is approximately $1 / 1,000$ person-years, $10 / 1,000$ person-years in general practice, and 30/1,000 person-years in the general population [38]. At the population level, "therapies' include patient and healthcare professional education, guidelines, screening programs, lifestyle interventions, referral pathways, and information systems. Although specialty multidisciplinary clinics manage a smaller population, those patients place a disproportionate burden on non-elective services and health system resources.

Table 3. Syncope and pre-syncope rates in treatment arms of randomized controlled trials.

\begin{tabular}{lccccc}
\hline Authors, years, trial & $\begin{array}{c}\text { Patients/ } \\
\text { /Centers (n) }\end{array}$ & Intervention & Follow-up & $\begin{array}{c}\text { Syncope } \\
\text { recurrence }\end{array}$ & Pre-syncope \\
\hline van Dijk et al., 2006 [17] PC Trial & $223 / 15$ & Counterpressure & 14 months & $32 \%(31 / 98)$ & $83 \%(81 / 98)$ \\
Duygu et al., 2008 [42] & $82 / 1$ & Orthostatic & 1 year & $37 \%(15 / 41)$ & - \\
Raviele et al., 1999 [9] VASIS & $126 / 20$ & Etilefrine & 262 days & $24 \%(15 / 63)$ & $41 \%(26 / 63)$ \\
Sheldon et al., 2006 [3] POST & $208 / 14$ & Metoprolol & 1 year & $36 \%(38 / 107)$ & - \\
Theodorakis et al., 2006 [41] & $96 / 1$ & Fluoxetine & 6 months & $9 \%(3 / 32)$ & $12 \%(4 / 32)$ \\
Connolly et al., 2003 [1] VPS II & $100 / 15$ & Pacing & 6 months & $33 \%(16 / 48)$ & $96 \%(46 / 48)$ \\
Brignole et al., 2012 [34] ISSUE-3 & $77 / 29$ & Pacing & 2 years & $25 \%$ & - \\
\hline
\end{tabular}




\section{Direction for clinical services and research}

Effective treatment of NCS requires a multidimensional strategy addressing all sections of the population. The community burden and prognosis needs to be defined, along with simple cost-effective strategies for diagnosis, screening, and risk stratification. The impact of brief educational and behavioral interventions in primary care should be investigated. Linked data systems and registries will prove invaluable in understanding transitions between primary care, ED attendances and unplanned hospitalizations. Rapid access to records of previous investigations and diagnoses would assist practitioners in reassuring patients, avoid hospitalizations, and hopefully alleviate maladaptive fears and beliefs. Standardized diagnostic criteria and therapeutic guidelines are equally important.

Randomization, blinding, and placebo or inactive comparators are essential in future studies to reduce bias. Optimal non-pharmacological therapy is equally important, to reduce confounding by simultaneous lifestyle change. All studies should measure symptom severity, generic and disease specific QOL. Generic instruments permit comparisons across populations and economic analysis (e.g. SF-36) [14, 39]. Disease-specific measures assess the impact on daily living and facilitate comparisons between studies in patients with the same disease (e.g. SFSQ and ISQL) [14, 27, 39]. Efforts should focus on understanding the mechanisms of psychological and social impairment through qualitative research. The applicability and cost-effectiveness of individual cognitive behavioral therapy, group therapy, and standardized educational material should be investigated.

Clearer definition of phenotypes and physiology is required. Obtaining a symptom rhythm correlation with burgeoning patch and ICM technology assists in defining normal as much as abnormal rhythm. Early vasoconstrictor studies examined patients with a vasodepressor provocation response, many of whom suffer bradyarrhythmia during spontaneous episodes [31]. Excluding these patients may improve the overall effectiveness of vasoconstrictors.

Acknowledging methodological limitations, midodrine demonstrates therapeutic potential with a weighted mean relative risk reduction of $62 \%$ for syncope in 5 prospective controlled studies [40]. The Prevention Of Syncope Trial IV (POST IV) multicenter randomized controlled trial is currently comparing midodrine to placebo in patients with recurrent vasovagal syncope diagnosed according to the Calgary Syncope Symptom Score [40]. The SSRIs also merit revisiting, particularly in conjunction with more detailed QOL and psychological assessment. The preliminary studies had insufficient sample size to demonstrate efficacy [41], but consistent direction and magnitude of effect in meta-analysis.

\section{A lesson from hypertension clinic}

The modern management of hypertension has shifted from maximal doses of a single agent to comprehensive lifestyle recommendations and multidrug strategies to address the many physiologic contributors to hypertension. NCS is in effect "Hypotension Clinic", but even more challenging because events are paroxysmal. Thus, expecting to address recurrent NCS with a single drug intervention is akin to the treatment of hypertension from 30 years ago. Multiple elements of blood pressure generation, regulation and alteration are likely to be required to effectively manage a troublesome case. Despite a lack of compelling evidence, the authors advocate a multidimensional approach to this challenging problem that focuses on:

1. Increasing blood pressure reserve.

2. Recognizing triggers and responding promptly.

3. Minimizing pharmacologic interventions that focus on nuances of patients and their physiology.

4. On-going enrollment in clinical trials to better define effective therapies.

\section{Conclusions}

Much priority has been assigned to better understanding the pathophysiology of NCS in order to match phenotypes and therapies. Aside from the limited efficacy to date, the complexity of personalized medicine adds many challenges including generalizability, cost-effectiveness, and implementation. A simpler approach is to deliver non-pharmacological interventions in the broadest sense to the broadest population. Exploring patient fears, misconceptions and behaviors may equally improve symptoms and morbidity. Finally, pharmacologic and device interventions should be used humbly and sparingly, with attempts to personalize therapies with "eyes wide open" regarding the weak evidence to support their use.

Conflict of interest: None declared 


\section{References}

1. Connolly SJ, Sheldon R, Thorpe KE et al. Pacemaker therapy for prevention of syncope in patients with recurrent severe vasovagal syncope: Second Vasovagal Pacemaker Study (VPS II): A randomized trial. JAMA, 2003; 289: 2224-2229.

2. Raviele A, Giada F, Menozzi C et al. A randomized, double-blind, placebo-controlled study of permanent cardiac pacing for the treatment of recurrent tilt-induced vasovagal syncope. The vasovagal syncope and pacing trial (SYNPACE). Eur Heart J, 2004; 25: 1741-1748.

3. Sheldon R, Connolly S, Rose S et al. Prevention of Syncope Trial (POST): A randomized, placebo-controlled study of metoprolol in the prevention of vasovagal syncope. Circulation, 2006; 113: 1164-1170.

4. Romme JJ, Reitsma JB, Black CN et al. Drugs and pacemakers for vasovagal, carotid sinus and situational syncope. Cochrane Database Syst Rev, 2011: CD004194.

5. Vyas A, Swaminathan PD, Zimmerman MB, Olshansky B. Are treatments for vasovagal syncope effective? A meta-analysis. Int J Cardiol, 2013; 167: 1906-1911.

6. Sud S, Massel D, Klein GJ et al. The expectation effect and cardiac pacing for refractory vasovagal syncope. Am J Med, 2007; 120: 54-62.

7. Nijjer SS, Pabari PA, Stegemann B et al. The limit of plausibility for predictors of response: application to biventricular pacing. J Am Coll Cardiol Cardiovasc Imag, 2012; 5: 1046-1065.

8. Sheldon RS, Morillo CA, Klingenheben T, Krahn AD, Sheldon A, Rose MS. Age-dependent effect of beta-blockers in preventing vasovagal syncope. Circ Arrhythm Electrophysiol, 2012; 5: 920-926.

9. Raviele A, Brignole M, Sutton R et al. Effect of etilefrine in preventing syncopal recurrence in patients with vasovagal syncope: A double-blind, randomized, placebo-controlled trial. The Vasovagal Syncope International Study. Circulation, 1999; 99: 1452-1457.

10. Moya A, Sutton R, Ammirati F et al. Guidelines for the diagnosis and management of syncope. Eur Heart J, 2009; 30: 2631-2671.

11. Sheldon R, Morillo C, Krahn A. Management of vasovagal syncope: 2004. Expert Rev Cardiovasc Ther, 2004; 2: 915-923.

12. Claydon VE, Hainsworth R. Salt supplementation improves orthostatic cerebral and peripheral vascular control in patients with syncope. Hypertension, 2004; 43: 809-813.

13. Natale A, Geiger MJ, Maglio C et al. Recurrence of neurocardiogenic syncope without pharmacologic interventions. Am J Cardiol, 1996; 77: 1001-1003.

14. Romme JJ, Reitsma JB, Go-Schon IK et al. Prospective evaluation of non-pharmacological treatment in vasovagal syncope. Europace, 2010; 12: 567-573.

15. Brignole M, Croci F, Menozzi C et al. Isometric arm counter-pressure maneuvers to abort impending vasovagal syncope. J Am Coll Cardiol, 2002; 40: 2053-2059.

16. Krediet CT, van Dijk N, Linzer M, van Lieshout JJ, Wieling W. Management of vasovagal syncope: controlling or aborting faints by leg crossing and muscle tensing. Circulation, 2002; 106: 1684-1689.

17. van Dijk N, Quartieri F, Blanc JJ et al. Effectiveness of physical counterpressure maneuvers in preventing vasovagal syncope: The Physical Counterpressure Manoeuvres Trial (PC-Trial). J Am Coll Cardiol, 2006; 48: 1652-1657.
18. Deharo JC, Jego C, Lanteaume A, Djiane P. An implantable loop recorder study of highly symptomatic vasovagal patients: The heart rhythm observed during a spontaneous syncope is identical to the recurrent syncope but not correlated with the head-up tilt test or adenosine triphosphate test. J Am Coll Cardiol, 2006; 47: 587-593.

19. Ammirati F, Colivicchi F, Santini M. Permanent cardiac pacing versus medical treatment for the prevention of recurrent vasovagal syncope: A multicenter, randomized, controlled trial. Circulation, 2001; 104: 52-57.

20. Ciani O, Buyse M, Garside R et al. Comparison of treatment effect sizes associated with surrogate and final patient relevant outcomes in randomised controlled trials: meta-epidemiological study. BMJ, 2013; 346: f457.

21. Wieseler B, Wolfram N, McGauran N et al. Completeness of reporting of patient-relevant clinical trial outcomes: comparison of unpublished clinical study reports with publicly available data. PLoS Med, 2013; 10: e1001526.

22. Moya A, Permanyer-Miralda G, Sagrista-Sauleda J et al. Limitations of head-up tilt test for evaluating the efficacy of therapeutic interventions in patients with vasovagal syncope: Results of a controlled study of etilefrine versus placebo. J Am Coll Cardiol, 1995; 25: 65-69.

23. Giada F, Silvestri I, Rossillo A, Nicotera PG, Manzillo GF, Raviele A. Psychiatric profile, quality of life and risk of syncopal recurrence in patients with tilt-induced vasovagal syncope. Europace, 2005; 7: 465-471.

24. Santhouse J, Carrier C, Arya S, Fowler H, Duncan S. A comparison of self-reported quality of life between patients with epilepsy and neurocardiogenic syncope. Epilepsia, 2007; 48: 1019-1022.

25. Rose MS, Koshman ML, Ritchie D, Sheldon R. The development and preliminary validation of a scale measuring the impact of syncope on quality of life. Europace, 2009; 11: 1369-1374.

26. Rose MS, Koshman ML, Spreng S, Sheldon R. The relationship between health-related quality of life and frequency of spells in patients with syncope. J Clin Epidemiol, 2000; 53: 1209-1216.

27. Linzer M, Gold DT, Pontinen M, Divine GW, Felder A, Brooks WB. Recurrent syncope as a chronic disease: preliminary validation of a disease-specific measure of functional impairment. J Gen Intern Med, 1994; 9: 181-186.

28. van Dijk N, Boer KR, Wieling W, Linzer M, Sprangers MA. Reliability, validity and responsiveness of the syncope functional status questionnaire. J Gen Intern Med, 2007; 22: 1280-1285.

29. Newton JL, Kenny RA, Baker CR. Cognitive behavioural therapy as a potential treatment for vasovagal/neurocardiogenic syncope: A pilot study. Europace, 2003; 5: 299-301.

30. Brignole M, Menozzi C, Del Rosso A et al. New classification of haemodynamics of vasovagal syncope: beyond the VASIS classification. Analysis of the pre-syncopal phase of the tilt test without and with nitroglycerin challenge. Vasovagal Syncope International Study. Europace, 2000; 2: 66-76.

31. Brignole M, Sutton R, Menozzi C et al. Lack of correlation between the responses to tilt testing and adenosine triphosphate test and the mechanism of spontaneous neurally mediated syncope. Eur Heart J, 2006; 27: 2232-2239.

32. Moya A, Brignole M, Menozzi C et al. Mechanism of syncope in patients with isolated syncope and in patients with tilt-positive syncope. Circulation, 2001; 104: 1261-1267.

33. Moya A, Brignole M, Sutton R et al. Reproducibility of electrocardiographic findings in patients with suspected reflex neurally-mediated syncope. Am J Cardiol, 2008; 102: 1518-1523. 
34. Brignole M, Menozzi C, Moya A et al. Pacemaker therapy in patients with neurally mediated syncope and documented asystole: Third International Study on Syncope of Uncertain Etiology (ISSUE-3): A randomized trial. Circulation, 2012; 125: 2566-2571.

35. Flammang D, Church TR, De Roy L et al. Treatment of unexplained syncope: a multicenter, randomized trial of cardiac pacing guided by adenosine 5'-triphosphate testing. Circulation, 2012; 125: 31-36.

36. Brignole M, Donateo P, Tomaino M et al. Benefit of pacemaker therapy in patients with presumed neurally mediated syncope and documented asystole is greater when tilt test is negative: An analysis from the third International Study on Syncope of Uncertain Etiology (ISSUE-3). Circ Arrhythm Electrophysiol, 2014; 7: 10-16.

37. Ganzeboom KS, Mairuhu G, Reitsma JB, Linzer M, Wieling W, van Dijk N. Lifetime cumulative incidence of syncope in the general population: A study of 549 Dutch subjects aged 35-60 years. J Cardiovasc Electrophysiol, 2006; 17: 1172-1176.
38. Olde Nordkamp LR, van Dijk N, Ganzeboom KS et al. Syncope prevalence in the ED compared to general practice and population: A strong selection process. Am J Emerg Med, 2009; 27: 271-279.

39. van Dijk N, Sprangers MA, Colman N, Boer KR, Wieling W, Linzer M. Clinical factors associated with quality of life in patients with transient loss of consciousness. J Cardiovasc Electrophysiol, 2006; 17: 998-1003.

40. Raj SR, Faris PD, McRae M, Sheldon RS. Rationale for the prevention of syncope trial IV: assessment of midodrine. Clin Autonomic Res, 2012; 22: 275-280.

41. Theodorakis GN, Leftheriotis D, Livanis EG et al. Fluoxetine vs. propranolol in the treatment of vasovagal syncope: A prospective, randomized, placebo-controlled study. Europace, 2006; 8: 193-198.

42. Duygu H, Zoghi M, Turk U et al. The role of tilt training in preventing recurrent syncope in patients with vasovagal syncope: A prospective and randomized study. Pacing Clin Electrophysiol, 2008; 31: 592-596. 